\title{
De l'importance de la prévention des maladies nosocomiales principalement d'origine hydrique en hygiène hospitalière
}

\author{
J.-M. JADIN*, A. LAFONTAINE** \\ *Membre de l'Académie Royale des Sciences d'Outre-Mer \\ Chargé de cours à l'Université Catholique de Louvain
}

Chef de Service à l'Hôpital de Jolimont; rue Ferrer, 159, B-7 100 La Louvière, Belgique

** Membre de l'Académie Royale de Médecine, Membre de l'Académie Nationale de

Médecine de France, Professeur émérite à l'Université Catholique de Louvain,

Directeur honoraire de l'Institut d'Hygiène et d'Épidémiologie de Belgique

\section{Introduction}

En Belgique, la mise en place des programmes d'une politique de prévention a abouti, à partir des années 1970, à la création des Comités d'Hygiène Hospitalière et à la nomination de Médecins Hygiénistes Hospitaliers et d'Infirmiers Hygiénistes sur la base de qualifications particulières. Cette évolution a été poursuivie par deux Arrêtés Royaux publiés au Moniteur Belge en 1988 et 1989.

Deux autres Arrêtés Royaux datant des 20 et 28 novembre 1990 prévoient les modalités de financement de l'hygiène hospitalière.

Le recueil et l'évaluation des données hospitalières relatives aux infections nosocomiales doivent être collectées et envoyées à l'Institut d'Hygiène et d'Epidémiologie Louis Pasteur de Bruxelles. Ces données y sont traitées de façon strictement confidentielle. Les résultats de chaque hôpital sont communiqués uniquement à l'hôpital concerné. Un premier rapport de synthèse sur la surveillance des maladies nosocomiales dans les unités de soins intensifs publié en janvier 1997 [Hanique G.] montre l'importance de la question.

\section{Définitions}

Une infection nosocomiale est une maladie infectieuse identifiable soit par la présence de bactéries, de parasites, d'agents de mycoses ou de prions. Elle est cliniquement identifiable. Cette maladie se contracte notamment en milieu hospita- 
lier. Elle peut concerner aussi bien un patient qu'un membre du personnel soignant dans ses activités hospitalières.

Le terme nosocomial diffère nettement du terme iatrogène qui comporte la notion d'erreur médicale, de négligence ou d'accident. A titre d'exemple, une pneumopathie survenant à la suite d'une fausse déglutition chez un patient immunodéprimé est une affection nosocomiale. Par contre une infection bronchique due à une maladresse lors d'une bronchoscopie est une infection iatrogène.

Dans tout hôpital, du fait de la nature même de leurs activités et de leurs structures, ce sont surtout les services d'urgence et celui de réanimation, qui représentent les plus hauts risques d'infection nosocomiale. Il ne faut cependant pas croire que les infections nosocomiales soient le privilège des services d'urgence et de réanimation ; la médecine interne, la chirurgie, la pédiatrie, la gynécologie obstétricale, par exemple, ont aussi leur part dans le problème. D'une manière générale, deux facteurs sont surtout à prendre en considération: la rupture des barrières physiques de protection, c'est-à-dire des barrières cutanéomuqueuses et la gravité des défaillances viscérales, immunitaires ou autres qui altèrent l'état général du patient.

Une notion à ne pas perdre de vue est celle du délai d'acquisition. Il correspond généralement au délai d'incubation minimum d'une infection aiguë provoquée par un germe à croissance rapide, c'est-à-dire habituellement $48 \mathrm{~h}$. Mais ce délai est raccourci lorsqu'il existe une relation patente entre un geste médical et l'infection du patient. L'exemple classique est la pneumopathie infectieuse après le recours à une instrumentation endoscopique compliquée chez un patient non à jeun et vomissant, mais qui doit être opéré en urgence. Ce patient présente des risques certains de pneumopathie nosocomiale.

Lorsqu'il y a eu pose de matériel étranger, prothèse valvulaire ou prothèse de col de fémur par exemple, le délai d'apparition d'une infection nosocomiale peut s'étendre sur un an ou plus.

Les plus longs délais d'apparition d'infection nosocomiale sont constitués par ne fut ce qu'une seule injection d'hormone de croissance d'origine humaine ou par une transplantation de dure-mère. On estime qu'il faut un délai de plusieurs années, si risque il y a, pour voir apparaître chez les patients ainsi traités, une maladie de Creutzfeldt-Jakob [Veyssier P. et Domart Y., 1996].

Le risque d'apparition d'une infection nosocomiale a tendance à s'accroître avec la durée d'hospitalisation.

Certains distinguent les infections nosocomiales endogènes, avec comme point de départ la flore cutanée ou intestinale du patient lui - même, des infections exogènes causées par des micro-organismes présents dans l'environnement du patient. En fait, ces deux flores se mélangent très vite, ce qui diminue l'intérêt pratique de cette distinction. 


\section{Description des Sources et des Voies de Contamination}

La diversité des sources et des voies de contamination dont la liste ci-après n'est pas exclusive, justifie que l'on prenne des mesures tant sur le plan de la prévention que sur le plan des actions à entreprendre si une infection nosocomiale apparaît. Les données de la littérature confirment cette position. Des centaines, des milliers de travaux attestent la nécessité des contrôles.

C'est ainsi que, même si au départ, l'eau du réseau de distribution est bactériologiquement correcte, la qualité de cette eau peut facilement se dégrader à cause de la complexité des circuits, de la longueur des réseaux hospitaliers de distribution et des travaux éventuels de réparation dont ceux-ci peuvent être l'objet.

Mais la qualité de l'eau n'est pas la seule à être en cause et une maladie nosocomiale peut survenir après une injection apparemment anodine.

L'étude de la littérature montre qu'une maladie nosocomiale peut apparaître:

- 1. Par voie cutanéo-muqueuse. L'utilisation d'eau de lavage et de crèmes dermatologiques peut être à la source de transmissions de Pseudomonas aeruginosa chez et par des jeunes patients atteints de mucoviscidose [Bosshammer et al., 1995].

Kolmos (1993) décrit la contamination de grands brûlés par Pseudomonas aeruginosa par l'eau courante utilisée pour irriguer les brûlures lors de l'admission à l'hôpital. Ces patients ont développé une septicémie.

- 2. Par voie digestive avec des germes entéropathogènes comme Salmonella typhimurium, Escherichia coli, Campylobacter jejuni., Serratia marcescens, Pseudomonas aeruginosa, Morganella morganii..., ces germes étant d'emblée résistants à beaucoup d'antibiotiques [Das et al., 1996]. Par ailleurs, les infections dues à la présence de Clostridium difficile résistant d'emblée à un grand nombre d'antibiotiques, sont en recrudescence. Settle et Wilcox (1996) signalent qu'en Angleterre ce germe a été six fois plus fréquent en 1993 qu'en 1990. Il faut à celui-ci trois semaines d'incubation. Un âge avancé et l'usage préalable d'antibiotiques sont deux importants facteurs de risque d'acquérir une souche toxigène de Clostridium difficile.

Dans une étude très documentée, Hardalo et Edberg (1997) démontrent la présence de Pseudomonas aeruginosa, un germe ubiquitaire, dans certaines eaux de distribution dites potables, dans des eaux mises en bouteille et dans quelques aliments. Certains clones de cette bactérie possèdent des facteurs de virulence suffisants pour proliférer dans des organes préalablement altérés et être à l'origine de pneumonies chez des patients souffrant de mucoviscidose,... Les auteurs proposent que les législations idoines interdisent la présence de cette bactérie dans l'eau dite potable et surtout dans l'eau mise en bouteille. 
- 3. Par contamination par l'environnement. Un germe connu sous le nom de Burkholderia (Pseudomonas) cepacia est un endocommensal des bulbes de fleurs. Il est apporté au chevet des patients,- on pourrait dire offert aux patients,- avec les fleurs et il contamine tables, éviers et mains des manipulateurs surtout au moment des arrosages et du changement d'eau de ces fleurs. On connaissait son action phytopathogène, il est connu maintenant pour son rôle pathogène dans le syndrome cepacia, une septicémie associée à une pneumonie fulminante fatale pour environ $20 \%$ des patients infectés. Il s'agit de patients traités par des produits immunodépresseurs ou souffrant de mucoviscidose ou de granulomatose chronique [Govan et al.].

Par ailleurs, les dentistes, qui traitent des enfants atteints de mucoviscidose et dont les crachats sont colonisés par cette bactérie, peuvent en utilisant leurs fraises, disperser des souches de Burkholderia ( $P$ s.) cepacia dans tout le cabinet chirurgical dentaire. Mais le risque de contamination est faible pour des patients sains [Pankhurst et al., 1995].

Le grand danger de Burkholderia (Ps.) cepacia réside dans le fait qu'il est généralement d'emblée résistant à tous les antibiotiques d'usage courant.

- 4. Par aérosol microbien hydrique de bacilles à Gram négatif tels que Pseudomonas aeruginosa, Legionella pneumophila, Mycobacterium xenopi [Bennett S.N.,1994], M. kansasii..., et de bactéries Gram positif tels que les Staphylocoques à coagulase négative.

Toute altération de l'intégrité de parois des cellules épithéliales bronchiques représente une porte d'entrée notamment pour les Pseudomonas et les Staphylocoques chez des patients ventilés mécaniquement ou ayant eu un lavage bronchoalvéolaire ou une aspiration endotrachéale [Jebrak G. and Mangiapan, 1996].

Bergogne-Berezin et Towner (1996) ont montré le rôle des Acinetobacter, et surtout d'Acinetobacter baumannii dans les affections nosocomiales endémiques. Ces micro-organismes présents dans de très petits volumes d'eau, sont renfermés dans des microgouttelettes ce qui en augmente les risques de dispersion.

Campbell \& coll. (1996) ont relevé un grand nombre de pneumonies nosocomiales à bacilles Gram-négatif provoquées accidentellement chez des patients intubés.

Fagon et al. (1996) a attiré l'attention sur Staphylococcus aureus responsable d'un grand nombre d'infections chez des patients hyperventilés.

Bertz et Lambertzechovsky (1996) insistent sur le fait que des sinusites nosocomiales évoluant vers des pneumonies, peuvent avoir comme point de départ une intubation endotrachéale, avec une incidence plus élevée pour la voie nasotrachéale qu'orotrachéale. Les germes incriminés sont $S$. aureus, $P$. aeruginosa et $A$. baumannii. Le traitement de ces sinusites est basé sur le retrait de tous les tubes passant par le pharynx, l'utilisation de substances topiques décongestionnantes et un drainage puis un lavage du sinus maxillaire. Le bénéfice d'une antibiothérapie éventuelle reste controversé. 
- 5. Par de l'eau circulant dans des appareils de monitoring sous - pression en chirurgie à cœur ouvert chez des patients qui ont développé par après des septicémies multiples à Enterobacter cloacae, Pseudomonas aeruginosa, Klebsiella pneumoniae, Klebsiella oxytoca et Serratia marcescens. L'enquête a démontré que cette contamination par l'eau aurait été due à la mauvaise utilisation d'un spray destiné à désinfecter ces appareils [Rudnick J.R. et al., 1996].

- 6. Par voie naso-pharyngienne. Dans les piscines, l'eau est maintenue à une température de 25 à $30^{\circ} \mathrm{C}$ et est constamment enrichie de matières organiques d'origine humaine. Cela constitue un merveilleux milieu de culture pour des amibes du groupe Naegleria. De telles conditions privilégiées se retrouvent dans les piscines servant au traitement de physiothérapie. Un moyen permettant de trouver rapidement ces amibes, est de filtrer l'eau suspecte et d'incuber aussitôt le filtrat dans un milieu approprié à $45^{\circ} \mathrm{C}$. Cette dernière modalité permet d'éliminer rapidement les autres amibes libres de l'eau et les bactéries qui ne peuvent supporter cette température élevée, qui, par contre, est favorable à une croissance rapide de $N$. fowleri. Le fait d'observer des amibes dans l'eau équivaut, par ailleurs, à l'identification d'un témoin important de la présence de bactéries dans l'eau, bactéries qui, comme on l'a vu, sont surtout d'origine humaine.

Le traitement de ces eaux, par filtration ou adjonction de produits chimiques ou encore par remplacement partiel ou total de l'eau, se fait habituellement de façon continue mais pas en fonction de critères réels tels que le nombre de nageurs par heure.

Grattard et al., 1996, ont étudié l'eau des bains ou des piscines de rééducation et de physiothérapie. De nombreux micro-organismes se développent dans différents «nids» microbiens.

La contamination s'explique facilement quand on sait que $N$. fowleri est présent dans de nombreux lacs et rivières [Cerva, 1971; Chang, 1971; Jadin, 1987]. De Jonckheere, 1979, a recherché la présence d'amibes dans seize piscines, en Belgique : $43.6 \%$ de ses isolements contenaient des Acanthamoeba dont $70 \%$ se sont montrées pathogènes pour la souris; $7.3 \%$ renfermaient des Naegleria différentes de $N$. fowleri. Le même auteur [De Jonckheere, 1982], étudiant la flore microbiologique renfermée dans des piscines d'hydrothérapie hospitalière, y a trouvé de nombreuses amibes thermophiles telles que $N$. lovaniensis dont la présence est souvent associée celle de $N$. fowleri. Cependant il n'a jamais pu observer cette dernière amibe.

- 7. Par utilisation d'eau désionisée [Farrington M. et al., 1996].

L'eau passe à travers des billes de résine sphériques échangeuses d'ions. L'eau échange ses ions contre ceux fixés sur la résine. Les avantages sont l'élimination d'ions, ce qui déminéralise efficacement l'eau, et le caractère régénérable de la résine. Les inconvénients principaux sont que cette méthode n'élimine ni les 
particules, ni les micro-organismes, ni les matières organiques. De telles eaux sont utilisées comme eau sanitaire, et comme eau à utiliser au début de chaînes d'hémodialyse ou de chaîne de production pour les laboratoires. Mais il importe en tous cas de purifier ces eaux par filtration en fonction de leur destination.

- 8. Par des cathéters d'hémodialyse dans un service de pédiatrie où de nombreux enfants ont fait des septicémies multiples à Enterococcus fecalis, Enterobacter cloacae, Staphylococcus epidermidis, Klebsiella pneumoniae. Le point de départ était souvent la veine sous-clavière à l'endroit de pose du cathéter [Hymes LC. et al., 1996].

Le rôle des cathéters est aussi souligné par Duggan et al. (1996) dans un groupe de patients souffrant de maladies cancéreuses $(30 \%)$, ayant un traitement immunosuppresseur ( $27 \%$ ) ou souffrant d'une pathologie cardiaque.

Le lavage et la désinfection de la peau sont souvent insuffisants. Dans de nombreux cas, la peau est considérée comme la source d'entrée de germes qui s'accolent momentanément à la paroi du cathéter. Ce mode d'infection représenterait aux USA $90 \%$ des 50.000 à 100.000 cas d'infection nosocomiale répertoriés chaque année [Adal K.A. and Farr B.M., 1996].

Les infections sur cathéter sont, en pédiatrie, une des premières causes d'infections nosocomiales. L'usage de cathéters veineux centraux doit se faire dans des conditions strictes de stérilité chirurgicale, car les risques d'infections sont fréquents.

- 9. Par le contact avec les mains des anesthésistes qui préfèrent parfois poser les cathéters mains nues pour mieux apprécier les voies d'abord veineuses. Comme ils ont lavé, au préalable, leurs mains, ils ne se rendent pas toujours compte que ce geste renouvelle le flore cutanée du patient [Fukada T. et al., 1966].

L'observation de la fréquence du lavage des mains de la part des anesthésistes et du personnel qualifié est très riche en enseignements et met en évidence que le nombre de lavages par jour et par acte est généralement très insuffisant par rapport aux fréquences de lavages proposées [Larson, 1988].

L'eau dite stérile des salles d'opération n'a pas toujours cette qualité [Petithory J.C. \& al., 1984].

Malavaud et Marty (1997) ont publié une intéressante réflexion sur la question, tant pour les précautions classiques que pour certaines mesures spécifiques, notamment en ce qui concerne les désinfections.

- 10. Nous ajouterons, par simple humour, les accidents survenus chez des patients gravement brûlés, et aspergés d'eau bénite à leur arrivée à l'hôpital. 
En fait, l'eau bénite est une eau stagnante laissée à l'air, sans protection, et dans laquelle de nombreuses personnes plongent leurs doigts qui ne sont pas toujours des modèles de propreté.

Dans des échantillons de cette eau, ont été isolées des souches de Pseudomonas aeruginosa, Escherichia coli, Enterobacter sp. et Candida sp. [Rees J.C., Allen K.D., 1996].

- 11. Par contact avec le bio-film constitué par certaines bactéries particulièrement Pseudomonas et Legionella pneumophila.

Les bactéries ont le pouvoir d'adhérer facilement à des supports inertes et attendent des conditions favorables de température et d'humidité pour s'y développer.

Bactéries vivantes, cadavres de bactéries et particules du support peuvent former un bio-film sur le sol, les surfaces de travail, les surfaces internes des circuits de ventilation ou des canalisation d'eau. Ce bio-film représente dans tout l'hôpital un réservoir où les patients fragiles peuvent se contaminer par la peau et les plaies, via le matériel utilisé ou par voie aérienne sous forme d'aérosols.

Le staphylocoque doré est fréquemment présent sur le textile et le mobilier. Pseudomonas est un des germes opportunistes le plus fréquemment isolé sur les surfaces humides d'autant qu'elles sont à température ambiante, telles les matériels sanitaires.

Dans la littérature, une attention toute particulière est accordée actuellement à Legionella pneumophila [Gahm-Hansen B. et al., 1995; Linde H.G. \& al., 1995]. Cette bactérie fait partie des bactéries que l'on isole de plus en plus souvent dans les réseaux de distribution d'eau des hôpitaux. Les Legionella envahissent les endroits corrodés des tuyauteries, les dispositifs d'absorption des chocs de pression [Memish Z.A., \& al., 1992], les humidificateurs d'air, les appareils producteurs d'aérosols. Elles affectionnent particulièrement les eaux à $35-45^{\circ} \mathrm{C}$. D'après Nahapetian \& al. (1991) la présence d'amibes libres favoriserait leur multiplication.

\section{Comment remédier à ces risques potentiels?}

Outre les mesures classiques d'hygiène (lavages énergiques et systématiques des mains, utilisation d'antiseptiques adéquats, emploi de matériel à usage unique, désinfection chimique du matériel sanitaire), il y a lieu de mener une réflexion sur la qualité de l'eau. Il faut connaître les circuits des réseaux de distribution d'eau de l'hôpital et des systèmes de d'humidification de l'air afin de pouvoir repérer les niches écologiques de certaines bactéries ou amibes de l'eau.

Il faut pouvoir recourir à certaines méthodes d'analyse suffisamment performantes que pour pouvoir comparer les bactéries de l'environnement avec celles isolées chez des patients atteints de maladies nosocomiales. 
Ces analyses peuvent consister en caractères de culture des souches, comparaison de la sensibilité des souches aux antibiotiques, sérotypage [Edelstein P.H., 1988], sensibilité aux bactériophages, typage biochimique, étude de l'ADN bactérien, [Luck \& al., 1995; Marrie T.J. \& al., 1995]...

Ces études permettront de réagir et de prendre les mesures nécessaires pour assurer aux différents services de l'hôpital une eau de qualité.

En fait, il faudrait scinder les différents usages de l'eau en fonction des besoins sanitaires, alimentaires, médicaux ou techniques et réserver l'eau microbiologiquement traitée aux patients présentant un haut risque infectieux dans les services de réanimation, néonatalogie, chirurgie, service des brûlés, hématologie et oncologie.

Parmi les procédures de maîtrise de la qualité de l'eau distribuée dans l'hôpital, citons:

- L'étude du réseau: élimination des espaces morts, remplacement des tuyaux corrodés,... Tout travail sur le réseau sera suivi d'un contrôle microbiologique [Mermel L.A. \& al., 1995].

- l'entretien régulier de la robinetterie, les purges qui éliminent les stagnations. Lorsqu'un contrôle microbiologique indique que le réseau est colonisé par un germe opportuniste comme Pseudomonas sp. ou Legionella, un choc thermique ou un choc chloré peuvent parfois résoudre le problème [Darelid J. \& al., 1994; Ezzeddine H. \& al., 1989 ; Muraca P.W. \& al., 1988; Prodinger W.M., 1994 ; Schultze-Robbecke R., 1990].

- Les dispositifs de traitement d'eau permettant d'obtenir une eau microbiologiquement traitée seront réservés aux services où les risques infectieux sont majeurs. Les meilleurs systèmes sont les systèmes de microfiltration soit terminale sur robinet, soit à un poste de filtration en amont de la robinetterie. Il faut veiller au remplacement des cartouches filtrantes usagées et à la maintenance de ces filtres.

Les lampes à rayons UV sont peu efficaces et coûteuses. La chloration permet aussi la production d'une eau stérile mais ce procédé exige d'être bien maîtrisé. L'application en continu d'une chloration avec un taux de chlore libre de 2 à 6 ppm (les Legionella, p. ex., sont relativement résistantes au chlore) est parfois utilisée. Il ne faut cependant pas oublier le risque de corrosion des conduits représentant de nouveaux nids microbiens potentiels, la formation de composés halogéniques organiques réputés carcinogènes et les problèmes d'interaction du chlore avec le principe actif de certains produits.

\section{Conclusions}

L'hygiène hospitalière se trouve confrontée quotidiennement au problème des infections nosocomiales: ce problème prend de plus en plus d'ampleur et comporte des responsabilités morales, scientifiques et budgétaires. 
Des mesures s'imposent en impliquant la responsabilité des scientifiques et des économistes. Les médecins spécialistes en biologie clinique doivent disposer de moyens financiers leur permettant de faire les recherches adéquates pour contrôler l'apparition de maladies nosocomiales, les endiguer et les combattre efficacement.

De telles mesures impliquent que les responsables puissent rester attentifs à la question et donc que des crédits indispensables et suffisants soient disponibles pour assurer à la fois les recherches fondamentales de routine et la surveillance quotidienne du milieu hospitalier pour éviter l'apparition inattendue et brutale de pathologies nosocomiales. nique.

Il devrait s'agir d'un budget différent du forfait hospitalier de biologie cli-

Ce forfait ne devrait jamais être l'objet de mesures aveugles d'économies. L'apparition brutale de pathologies nosocomiales est toujours la source de dépenses financières qu'aucun économiste, si bon soit-il, ne parviendra jamais à estimer correctement.

Il faut rechercher l'efficacité maximale si possible au moindre coût. Une bonne analyse économique démontrerait à l'évidence que la prévention des infections nosocomiales pourrait éviter des traitements onéreux sans parler du bénéfice du capital santé et moral du patient.

\section{BIBLIOGRAPHIE}

Adal K.A. and Farr B.M., 1996, Central venous catheter - related infections. A Review. Nutrition, 12, 208-213.

Bennett S.N., Peterson D.E., Johnson D.R., Hall W.N., Robinson-Dunn B., Dietrich S., 1994, Bronchoscopy-associated Mycobacterium xenopi pseudoinfections. Am. J. of Resp. \& Crit. Care Med., 150, 245-250.

Bergogne-Berezin E. and Towner K.J., 1996, Acinetobacter spp., as nosocomial pathogens - microbiological, clinical, and epidemiological features. Clin. Microbiol. Rev., 9, 148.

Bert F., Lambertzechovsky N., 1996, Sinusitis in mechanically ventilated patients and its role in the pathogenesis of nosocomial pneumonia. Europ.J. of Clin. Microbiol. and Infect. Dis., 15, 533-544.

Bosshammer J, Fiedler B., Gudowius P., von der Hardt H., Romling U., Tummler B., 1995, Comparative hygienic surveillance of contamination with pseudomonads in a cystic fibrosis ward over a 4-year period. J. of Hosp. Inf., 31, 261-274.

Campbell G.D., Niederman M.S., Broughton W.A., Craven D.E., Fein A.M., Fink M.P., Gleeson K., Hornick D.B., Lynch J.P., Mandell L.A., Mason C.M., Torres A. Wunderink R.G., 1996, Hospital-acquired pneumonia in adults - Diagnosis, assessment of 
severity, initial antimicrobial therapy, and preventive strategies - a consensus statement. Am. J. of Resp. \& Crit. Care Med., 153, 1711-1725.

Cerva L., 1971, Studies of limax amoeba in a swimming pool, Idrobiologia, 38, 141-161.

Chang F.Y., Jacobs S.L., Colodny S.M., Stout J.E., Yu V.L., 1996, Nosocomial Legionnaires'disease caused by Legionella pneumophila serogroup 5 : laboratory and epidemiologic implications. J. of Inf. Dis., 174, 1116-1119.

Chang S. L., 1971, Small free-living amoebae cultivation, quantitation, identification, classification, pathogenesis and resistance. Curr. Top. Comp. Pathobiol., 1, 201-254.

Darelid J., Bengtsson L., Gastrin B., Hallander H., Lofgren S., Maimvall B.E., OlinderNielsen A.M., Thelin A.C., 1994, An outbreak of Legionnaires'disease in a Swedish hospital. Scand. J. of Infect. Diseases, 26, 417-425.

Das A.S., Mazumder D.N., Pal D., Chattopadhyay U.K., 1996, A study of nosocomial diarrhea in Calcutta. Ind. J. of Gastroenterol., 15, 12-13.

De Jonckheere J.F., 1979, Pathogenic free-living amoebae in swimming pools: survey in Belgium. Ann. Microbiol. (Inst. Pasteur), 130 B, 205.

De Jonckheere J.F., 1982, Hospital hydrotherapy pools treated with ultra violet light: bad bacteriological quality and presence of thermophilic Naegleria. J. Hyg., 88,205-214.

Duggan J.M., Golstein S.J., Chenoweth C. E., Kauffman C.A., Bradley S.F., 1996, Achromobacter xylosoxidans bacteremia- report of four cases and review of the litterature. Clin. Infect. Dis., 23, 569-576.

Edelstein P.H., 1988, Nosocomial Legionnaires'disease: a global perspective. J. of Hosp. Infect., 11 suppl A, 182-188.

Ezzeddine H., Van Ossel C., Delmée M., Wauters G., 1989, Legionella spp. in a hospital hot water system : effect of control measures. J. of Hosp. Infect., 13,121-131.

Fagon J.Y., Trouillet J.L., Chastre J., 1996, Intensive care units-acquired pneumonia. Presse Méd., 25, 1441-1446.

Farrington M., Matthews I., Foreman J., Caffey E., 1996 Bone graft contamination from a water de - ionizer during processing in a bone bank. J. of Hospit. Infect., 32, 61-64.

Fukada T., Tachibana C., Tsugazaki Y., Saton K., Furuya., Ghe Y., 1996, Bacterial contamination of anesthesiologists'hands and the efficacy of handwashing. Masui - Japanese J.. Anesthesiol. 45, 1026-1030.

Gahm-Hansen B., Uldum S.A.., Schmidt J., Nielsen B., Birkeland S.A., Jorgensen K.A., 1995, Nosocomial Legionella pneumophila infection in a nephrology departement (danish). Ugeskrift for Laeger, 157, 590-594.

Govan J.R.W., Hughes J.E., Vandamme P., 1996, Burkholderia cepacia - medical, taxonomic and ecological issues. J. of Med. Microbiol. 45, 395-407. 
Grattard F., Berthelot P., Reyrolle M., Ros A., Etienne J., Pozzetto B., 1996, Molecular typing of nosocomial strains of Legionella pneumophila by arbitrary primed PCR. J. Clin. Microbiol., 34, 1595-1598.

Hanique G., 1997, Surveillance des Infections nosocomiales aux Soins Intensifs. Résultats Nationaux. 1er Rapport de Synthèse de janvier 1996 à juin 1996. 1-23 et 3 annexes.

Hardalo C., Edberg S.C., 1997, Pseudomonas aeruginosa - Assessment of risk from drinking water. Critical Rev. in Microbiol., 23, 47-75.

Hymes L.C., Warschaw B.L., Clowers B., Newsome P., Keyserling H. L, 1996, Bacteremia in a pediatric hemodialysis unit secondary to Enterococcus fecalis. Ped. Nephrol. 10, $55-57$.

Jadin J.B., 1987, in Infectious diseases. Color atlas monographs, 1 Amphizoic Amoeba, Human Pathology, 1, 1-23.

Jebrak G. and Mangiapan G., 1996, Nosocomial pneumonia, Presse Médicale, 25, 944-950.

Kolmos H.J., Thuesen B., Nielsen S.V., Lohmann M., Kristoffersen K., Rosdahl V.T., 1993, Outbreak of infection in a burns unit due to Pseudomonas aeruginosa originating from contaminated tubing used for irrigation of patients. J. of Hosp. Inf., 24,11-21.

Larson E., 1988, Guidline for use of topical antimicrobial agents. Am. J. Inf. Contr., 16, 253-266.

Linde H.J., Hengere A., Voggesberger E., Hecht J., Ereht W., Wolf H., 1995, Eradication of Legionella from warm watersystems. Documentation of our own experiences with thermal desinfection. Zentralblatt fur Hygiene and Umweltmedizin, 197, 441-451.

Luck P.C., KohlerJ., Maiwald M., Helbig J.H., 1995, DNA polymorphisms in strains of Legionella pneumophila serogroup 3 and 4 detected by macrorestriction analysis and their use for epidemiological investigation of nosocomial legionellosis. Appl. \& Environmental Microbiol., 61, 2000-2003.

Malavaud S., Marty N., 1997, Le risque infectieux au cabinet médical : une réalité à combattre. La Presse Méd., 26, n² 21, 1008-1012.

Marrie T.J., Johnson W., Tyler S., Bezanson G., Haldane D., Burbridge S., Joly J., 1995, Potable water and nosocomial Legionnaires'disease - check water from all rooms in which patient has stayed. Epidemiol. \& Infect., 114,267-276.

Memish Z.A., Oxley C., Contant J., Garber G.E., 1992, Plumbing system shock absorbers as a source of Legionella pneumophila. Am. J. of Infect. Contr., 20, 305-309.

Mermel L.A., Josephson S.L., Giorgio C.H., Dempsey J., Parenteau S., 1995, Association of Legionnaires'disease with construction: contamination of potable water?. Inf. Contr. \& Hosp. Epidemiol., 16, 76-81.

Nahapetian K., Challemel O., Beurtin D., Dubrou S., Gounon P., Squinazi F., 1991, The intracellular multiplication of Legionella pneumophila in protozoa from hospital plumbing systems. Res. In Microbiol., 142, 677-685. 
Pankhurst CL., Harrisson V.E., Philpott-Howard J., 1995, Evaluation of contamination of the dentist anf dental surgery environment with B. (Ps.) cepacia during treatement of children with cystic fibrosis, Int. J. of Paediatr. Dentist., 5, 243-247.

Petithory J.C., Barraud D., Escallier G., Cochet P., 1984, A propos de l'eau «stérile» des salles d'opération. Bull. Acad. Nat. Méd., 168, 255-261.

Prodinger W.M., Bonatti H., Allerberger F., Wewalka G., Harrison T.G., Aichberger C., Dierich M.P., Tiefenbrunner F., 1994, Legionella pneumonia in transplant recipients : a cluster of cases of eight years'duration. J. of Hosp. Infect., 26, 191-202.

Rees J.C., Allen K.D., 1996, Holy water, a risk factor for hospital acquired infection, J. of Hosp. Inf., 32, 51-55.

Rudnick J.R., Beck-Sague C.M., Anderson R.L., Schable M., Miller J.M., Jarvis W.R.., 1996, Gram-negative bacteremia in open-heart-surgery patients traced to probable tapwater contamination of pressure monitoring equipment. Inf. Contr. \& Hosp. Epidemiol., 17, 281-285.

Schulze-Robbeke R., Jung K.D., Pulmann H., Hundgeburth J., 1990, Sanitizing a hospital hot water system contaminated with Legionella pneumophila (german). Zentralblatt fur Hyg. Und Umweltmedizin, 190, 84-100.

Settle C.D. and Wilcox M.H., 1996, Review article - Antibiotic - induced Clostridium difficile infection, Alimentary Pharmacol. \& Therapeut., 10, 835-841..

Veyssier P. et Domart Y., 1996, Infections Nosocomiales, Ed. Masson, 1-158. 